\section{(C) OPEN ACCESS}

\title{
Clinical implications of early caudate dysfunction in Parkinson's disease
}

\author{
Jacopo Pasquini, ${ }^{\oplus 1,2}$ Rory Durcan, ${ }^{3}$ Louise Wiblin, ${ }^{3}$ Morten Gersel Stokholm, ${ }^{4}$ \\ Lynn Rochester, ${ }^{3,5}$ David James Brooks, ${ }^{3,4}$ David Burn, ${ }^{5,6}$ Nicola Pavese $^{3,4}$
}

\begin{abstract}
- Additional material is published online only. To view please visit the journal online (http://dx.doi.org/10.1136/ jnnp-2018-320157).

${ }^{1}$ Dipartimento di Fisiopatologia Medico-Chirurgica e dei Trapianti, University of Milan, Milan, Italy

${ }^{2}$ Department of Neurology Stroke Unit and Laboratory of Neuroscience, IRCCS Istituto Auxologico Italiano, Milan, Italy ${ }^{3}$ Institute of Neuroscience, Newcastle University, Newcastle upon Tyne, UK

${ }^{4}$ Department of Nuclear Medicine and PET Centre, Aarhus Universitetshospital, Aarhus, Denmark

${ }^{5}$ Newcastle upon Tyne Hospitals, NHS Foundation Trust, Newcastle upon Tyne, UK ${ }^{6}$ Faculty of Medical Sciences, Newcastle University, Newcastle upon Tyne, UK
\end{abstract}

\section{Correspondence to}

Professor Nicola Pavese, Institute of Neuroscience, Newcastle University, Newcastle upon Tyne NE1 7RU, Tyne and Wear, UK; Nicola.Pavese@ newcastle.ac.uk

JP and RD contributed equally.

Received 8 December 2018 Revised 26 February 2019 Accepted 15 April 2019 Published Online First 11 May 2019

\section{Check for updates}

(C) Author(s) (or their employer(s)) 2019. Re-use permitted under CC BY-NC. No commercial re-use. See rights and permissions. Published by BMJ.

To cite: Pasquini J, Durcan $R$, Wiblin L, et al. J Neurol

Neurosurg Psychiatry

2019:90:1098-1104.

\section{ABSTRACT}

Objective Although not typical of Parkinson's disease (PD), caudate dopaminergic dysfunction can occur in early stages of the disease. However, its frequency and longitudinal implications in large cohorts of recently diagnosed patients remain to be established. We investigated the occurrence of caudate dopaminergic dysfunction in the very early phases of PD $(<2$ years from diagnosis) using ${ }^{123}$ I-FP-CIT single photon emission CT and determined whether it was associated with the presence or subsequent development of cognitive impairment, depression, sleep and gait problems. Methods Patients with PD and healthy controls were identified from the Parkinson's Progression Markers Initiative (PPMI) database. We defined a clinically significant caudate dysfunction as ${ }^{123}$ I-FP-CIT binding $<-2$ SDs compared with the controls' mean and categorised three groups accordingly (no reduction, unilateral reduction, bilateral reduction). All statistical analyses were adjusted for mean putamen binding. Results At baseline, $51.6 \%$ of 397 patients had normal caudate dopamine transporter binding, $26.0 \%$ had unilateral caudate involvement, $22.4 \%$ had bilaterally impaired caudate.

Compared with those with a baseline normal caudate function, at the4-year follow-up patients with a baseline bilateral caudate involvement showed a higher frequency of cognitive impairment $(p<0.001)$ and depression $(p<0.001)$, and worse cognitive $(p<0.001)$, depression $(<0.05)$ and gait $(<0.001)$ ratings. Significant caudate involvement was observed in $83.9 \%$ of the population after 4 years (unilateral $22.5 \%$, bilateral $61.4 \%$ ).

Conclusions Early significant caudate dopaminergic denervation was found in half of the cases in the PPMI series. Baseline bilateral caudate involvement was associated with increased risk of developing cognitive impairment, depression and gait problems over the next 4 years.

\section{INTRODUCTION}

Caudate dopaminergic dysfunction is commonly seen in patients with established and advanced Parkinson's disease (PD) and plays a role in the pathophysiology of parkinsonian symptoms such as cognitive impairment, ${ }^{1-7}$ depression, ${ }^{8}$ REM sleep behaviour disorder (RBD) ${ }^{9}$ and gait problems. ${ }^{10} 11$ Conversely, both postmortem and in vivo imaging studies suggest that caudate function is preserved in the early stages of PD. In fact, dopaminergic neurons of the substantia nigra pars compacta $(\mathrm{SNc})$ degenerate in a selective pattern, with earliest and most severe loss occurring in the ventral tier projecting to the posterior putamen and tail of caudate, followed by the dorsal tier projecting to the head of caudate nucleus, the globus pallidus and the neocortex. ${ }^{12} 13$ Neuroimaging studies using single photon emission CT (SPECT) and PET dopaminergic tracers ${ }^{14-16}$ confirm that the dopaminergic deficit within the striatum is unevenly distributed with a more severe involvement of the posterior putamen and a relative sparing of the head of caudate nucleus. This asymmetrical posterior-to-anterior gradient of dopaminergic dysfunction is present from early disease stages and does not change substantially with disease progression. ${ }^{17} 18$

However, the occurrence of caudate involvement in the early stages of the disease and the clinical implications of such early caudate dysfunction (ie, the current or subsequent manifestation of cognitive impairment, depression, RBD, gait problems) have not been fully investigated in large cohorts.

\section{MATERIALS AND METHODS}

\section{Study design and participants}

In this longitudinal study, we analysed clinical ratings and $\mathrm{N}$ - $\omega$-fluoropropyl-2$\beta$-carbomethoxy-3 $\beta-\left(4-\left[{ }^{123}\right.\right.$ I] iodophenyl)nortropane $\left({ }^{123}\right.$ I-FP-CIT) SPECT data from a cohort of patients with early stage PD and healthy controls (HCs) recruited in the Parkinson's Progression Markers Initiative (PPMI), an ongoing multicentre, longitudinal study aiming to identify biomarkers of PD progression. Patients with PD were required to have a clinical diagnosis for 2 years or less, be untreated and show evidence of striatal dopamine transporter (DAT) deficit on SPECT imaging (study protocol available at https://www.ppmi-info.org/studydesign/research-documents-and-sops/). ${ }^{19}$

We performed three main analyses: (1) we evaluated caudate ${ }^{123}$ I-FP-CIT binding at baseline and follow-up in patients with PD and HCs. Patients with PD were categorised in three subgroups according to their baseline caudate binding compared with HCs (no reduction, unilateral reduction, bilateral reduction); (2) we assessed whether these three PD subgroups showed different baseline manifestations in terms of cognitive impairment, depression, RBD and gait problems; (3) we investigated whether the initial pattern of caudate dopaminergic dysfunction was able to predict worse outcomes and increased risk of developing cognitive, mood, sleep and gait problems at 4-year follow-up. 
At the time of our analysis, we retrieved 405 patients with PD and 177 HCs whose baseline assessments, including SPECT scan, motor and non-motor scores were available in the PPMI database (http://www.ppmi-info.org/data). At 4-year follow-up, clinical assessments were available for 328 patients and SPECT imaging for 267. Four-year follow-up clinical assessments were available for $151 \mathrm{HCs}$; no follow-up SPECT imaging was provided for HCs in the PPMI.

${ }^{123}$ I-FP-CIT SPECT striatal specific binding ratios (SBRs) and demographics and clinical variables at baseline and 4-year follow-up of all the study participants were downloaded from the PPMI database on 15 May 2018.

\section{Clinical evaluations}

PD motor disability was assessed with the Movement Disorder Society Unified Parkinson's Disease Rating Scale (MDS-UPDRS) and the Hoehn \& Yahr (H\&Y) scale. To assess differences in gait impairment between groups at baseline and 4-year follow-up, we calculated an index of gait severity by using the product of the patient's self-reported walking and balance score (subitem 2.12, MDS-UPDRS part II) and the freezing score (2.13, MDS-UPDRS part II) (Maximum score=16). All scores were collected in the OFF state.

Scores of the following non-motor scales were also obtained: Montreal Cognitive Assessment (MoCA), the 15-item Geriatric Depression Scale (GDS) and RBD Screening Questionnaire (RBDSQ). For each patient, one point was added to the MoCA unadjusted score of those who had 12 years of education or less, as indicated in the test original validation study ${ }^{20}$ and in the PPMI protocol. A GDS score $\geq 5$ was considered diagnostic for the presence of significant depressive features. ${ }^{21}$

Study participants were allocated to one of three cognitive categories (normal cognition, mild cognitive impairment (MCI), dementia) based on recommended criteria for dementia ${ }^{22}$ and $\mathrm{MCI}^{23}$ and as outlined in the PPMI protocol (see online supplementary material-section 1 ).

Last, the final clinical diagnosis at 4-year follow-up based on clinical judgement of the investigator was recorded to determine if the patient's diagnosis had changed compared with baseline.

Eight patients initially diagnosed as idiopathic PD were rediagnosed as a form of atypical parkinsonism: four had their diagnosis switched to multiple system atrophy (MSA), three to dementia with Lewy bodies (DLB), one to corticobasal degeneration (CBD). These patients were excluded from the main analysis and their neuroimaging features analysed separately. Therefore, a total of 397 patients at baseline and 323 patients at follow-up were included in the main analysis.

\section{${ }^{123}$ I-FP-CIT SPECT imaging protocol}

A detailed description is provided in online supplementary material-Section 2. Briefly, all subjects underwent ${ }^{123}$ I-FP-CIT SPECT scans during the screening visit and at follow-ups (patients with PD only) at their respective PPMI imaging centre, where standardised imaging protocols were used. Raw SPECT data were transferred back to the Institute for Neurodegenerative Disorders, New Haven, Connecticut, USA (PPMI Imaging Core), for processing and calculations of SBRs. On transfer to the PPMI imaging core, images were processed and normalised to standard Montreal Neurologic Institute (MNI) space so that all scans were in the same anatomical alignment. Next, the transaxial slice with the highest striatal binding was identified and the eight contiguous slices with highest striatal signal were then averaged to generate a single transaxial image. Regions of interest
(ROI) were then placed to sample the left (area $240 \mathrm{~mm}^{2}$ ) and right caudate (area $244 \mathrm{~mm}^{2}$ ), the left (area $412 \mathrm{~mm}$ ) and right putamen (area $408 \mathrm{~mm}^{2}$ ) and the occipital cortex (reference tissue, area $4500 \mathrm{~mm}^{2}$ ).

Count densities for each region were extracted and used to calculate SBRs for each of the four striatal subregions.

$$
S B R=(\text { target region/reference region })-1 \text {. }
$$

SBRs of caudate and putamen of each participant were downloaded from the PPMI database. SBR measures were rounded to the first two decimals. Mean caudate DAT SBR \pm SD $(95 \% \mathrm{CI})$ in controls was $2.96 \pm 0.60(2.88-3.06)$. The caudate signal was considered to be significantly abnormal in patients with PD if its level of ${ }^{123}$ I-FP-CIT binding fell two SD or more below the mean caudate binding of HCs at baseline (mean -2SDs=1.76). Three PD subgroups were then categorised as follows: those with no reduced ${ }^{123} \mathrm{I}$-FP-CIT binding in either caudate (PD-NC), those with reduced caudate binding in one caudate only (PD-UC) and those with bilaterally reduced caudate binding (PD-BC). Accordingly, we defined significant early caudate dysfunction a caudate ${ }^{123}$ I-FP-CIT binding < - 2 SDs compared with the controls' mean at the baseline SPECT acquisition.

\section{Statistical analysis}

Statistical interrogations were performed using the Statistical Package for the Social Sciences V.21 (SPSS 21). For descriptive analyses, means and SD were computed for continuous variables, and the $\chi^{2}$ test was used for categorical variables. Data were assessed for a normal distribution using Kolmogorov-Smirnov test. MoCA, GDS and RDBSQ ratings were normally distributed, gait index scores showed a non-normal distribution.

At baseline, between-group differences were investigated using analysis of variance followed by a posthoc test with Bonferroni correction for normally distributed data. At follow-up, betweengroup differences of normally distributed variables were investigated through analysis of covariance followed by a posthoc test with Bonferroni correction, holding putamen, age and years of education as covariates where appropriate. Non-normally distributed data were explored through a Kruskal Wallis test followed by Mann-Whitney U tests, and $\mathrm{p}$ values were adjusted through a Bonferroni correction. All multiple comparisons for categorical data at baseline and follow-up were assessed through a $\chi^{2}$ omnibus test followed by a standardised residuals analysis; $\mathrm{p}$ values were adjusted through a Bonferroni correction.

\section{RESULTS}

\section{Baseline}

The baseline analysis included 397 patients with PD (men/ women: 258/139, age: 61.7 \pm 9.7 years) and $177 \mathrm{HCs}$ (men/ women: $116 / 61$, age: $60.95 \pm 11.2$ years). In 397 patients with PD, normal ${ }^{123}$ I-FP-CIT binding in either caudate nuclei (PD-NC) was noted in $51.6 \%(n=205)$ of patients, $26.0 \%(n=103)$ had reduced tracer binding in one caudate only (PD-UC) and $22.4 \%$ $(n=89)$ had reduced tracer binding in both caudate nuclei (PD-BC) (figure 1A). No significant differences were found in the left/right distribution of the most affected caudate across the three PD subgroups. At baseline, all patients with a unilateral or bilateral caudate DAT reduction also had either a unilateral or bilateral putaminal reduction. More details about caudate and putamen involvement in individual patients are reported in online supplementary tables 1 and 2. Mean putamen SBR was significantly different across the three groups $(F=90.003, p<0.001)$, with PD-BC significantly lower than the other two groups, and PD-UC significantly lower than PD-NC. However, the caudate/ 

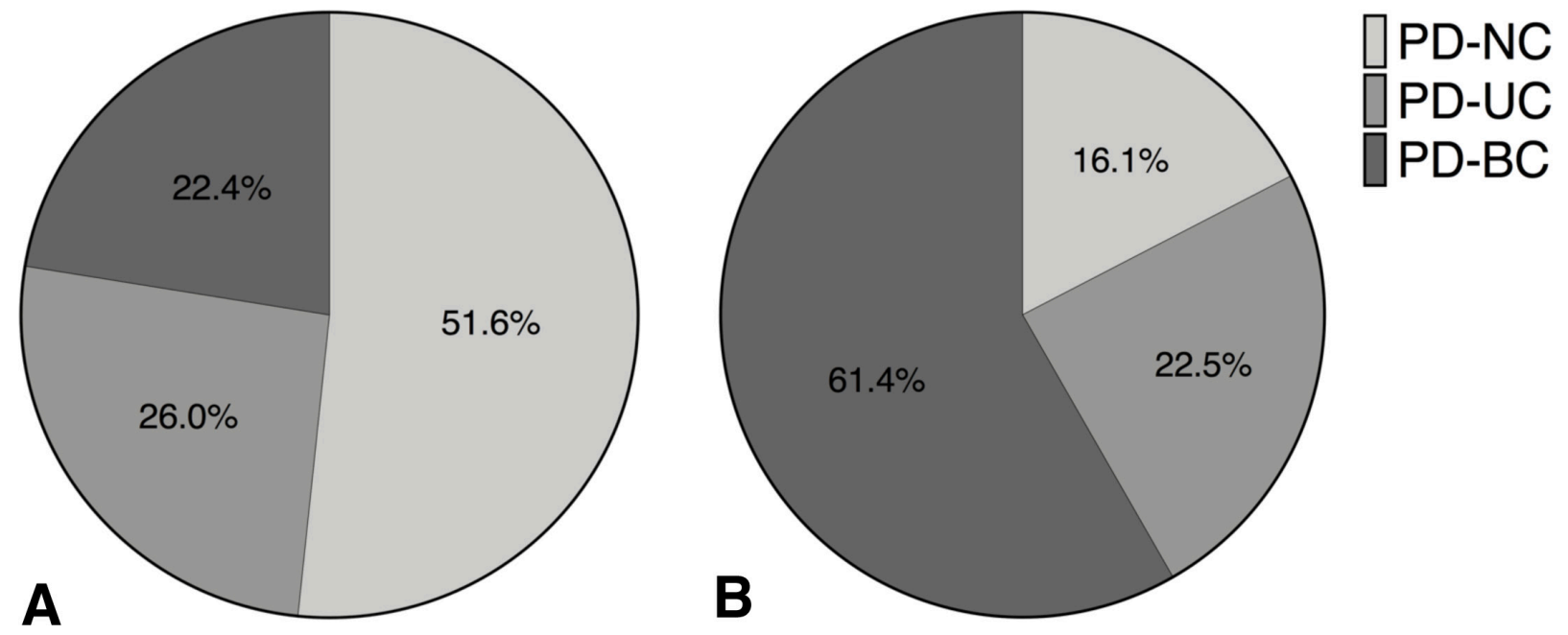

Figure 1 Pie chart representing the three groups of patients at baseline (A) and follow-up (B) classified according to their baseline caudate ${ }^{123}$ I-FP-CIT SPECT binding compared with healthy controls. Patients with Parkinson's disease (baseline, $n=397$; follow-up, $n=267$ ) were categorised into three groups based on their baseline caudate specific binding ratios compared with healthy controls: no reduced ${ }^{123} \mathrm{I}-\mathrm{FP}-\mathrm{CIT}$ binding in either caudate (PD-NC); ${ }^{123} \mathrm{I}-\mathrm{FP}-\mathrm{CIT}$ caudate binding reduced below -2 SDs of the controls' mean in one caudate only (PD-UC); ${ }^{123}$ I-FP-CIT caudate binding bilaterally reduced below -2 SDs of controls' mean (PD-BC). The percentage of each subgroup in the PD cohort is displayed on the corresponding pie slice. PD, Parkinson's disease.

putamen ratio, which provides an index of the posterior-to-anterior dopaminergic loss gradient, was not significantly different across the three groups. Age across the three PD subgroups and HCs was significantly different $(\mathrm{F}=3.657, \mathrm{p}<0.05)$, with the PD-BC group being older $(64.46 \pm 8.13$ years) than HCs $(60.95 \pm 11.20$ years) and the other two PD subgroups (PD-NC: $60.98 \pm 10.16$ years; PD-UC $60.63 \pm 9.76$ years). In order to assess if there was a significant age-related decline in caudate DAT availability in our control cohort, we compared mean caudate SBRs in three subgroups of controls classified according to their age $(50-59,60-69, \geq 70)$. No significant differences were found in mean caudate DAT binding across these groups $(\mathrm{F}=2.305, \mathrm{p}=0.103)$. In regard to gender, we did not find any difference in the distribution of males and females across the three groups PD-NC, PD-UC, PD-BC $\left(\chi^{2}=0.221, \mathrm{p}=0.895\right)$. We also did not find any significant difference between males and females in right, left and mean caudate DAT binding. The three PD subgroups did not differ significantly either in terms of disease duration or in motor symptoms as assessed through MDS-UPDRS III and H\&Y scale scores (table 1).

We then compared scores and frequencies of cognitive impairment, depression, RBD and gait problems between patients with PD and HCs, and between PD subgroups. All the three PD subgroups had a significantly lower mean MoCA score compared

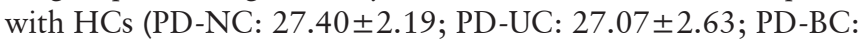
$27.30 \pm 2.31 ; \mathrm{HC}: 28.38 \pm 1.23 ; \mathrm{F}=12.171, \mathrm{p}<0.001)$. Across PD subgroups, there were no differences in MoCA scores. For $\mathrm{HCs}$, all scores were within the range for normal cognition (cutoff $<26$ ) as outlined in the PPMI protocol.

In terms of clinically significant depressive features as rated by a GDS score $\geq 5$, the frequency of these was higher in all PD subgroups (PD-NC: 11.2\%; PD-UC: 16.5\%; PD-BC: $18.0 \%$ ) compared with HC $(6.2 \%)\left(\chi^{2}=10.919, \mathrm{p}<0.05\right)$. There were no significant differences in the frequency of depressive symptoms or in GDS scores between PD subgroups.

A significant difference in mean RBDSQ score was seen between PD subgroups and HC group (PD-NC: 4.35 \pm 2.86 ; PD-UC: 4.29 \pm 2.50 ; PD-BC: 5.02 \pm 3.18 ; HC: 2.97 \pm 2.38 ; $\mathrm{F}=14.152 \mathrm{p}<0.001)$. There was no significant difference between PD subgroups in terms of RBD score.
There was no difference in self-reported gait impairment as measured by the gait index between the PD subgroups at baseline $(\mathrm{H}=3.924, \mathrm{p}=0.141)$.

No significant correlations were found between mean caudate ${ }^{123}$ I-FP-CIT binding and cognitive, mood, RBD and gait impairment ratings at baseline.

\begin{tabular}{|c|c|c|c|c|}
\hline & $\begin{array}{l}\text { Healthy } \\
\text { controls } \\
(\mathrm{n}=177) \\
\text { Mean } \pm S D\end{array}$ & $\begin{array}{l}\text { PD-NC } \\
(\mathrm{n}=205) \\
M e a n \pm S D\end{array}$ & $\begin{array}{l}\text { PD-UC } \\
(\mathrm{n}=103) \\
M e a n \pm S D\end{array}$ & $\begin{array}{l}\text { PD-BC } \\
(\mathrm{n}=89) \\
\text { Mean } \pm S D\end{array}$ \\
\hline Age, years & $60.95 \pm 11.20$ & $60.98 \pm 10.16$ & $60.63 \pm 9.76$ & $64.46 \pm 8.13^{*}$ \\
\hline Male/Female & $116 / 61$ & $131 / 74$ & $68 / 35$ & $59 / 30$ \\
\hline $\begin{array}{l}\text { Years of } \\
\text { education }\end{array}$ & $16.11 \pm 2.93$ & $15.75 \pm 2.92$ & $15.20 \pm 3.15$ & $15.42 \pm 2.97$ \\
\hline $\begin{array}{l}\text { Duration of } \\
\text { symptoms, } \\
\text { years }\end{array}$ & & $1.53 \pm 2.40$ & $1.55 \pm 1.63$ & $1.30 \pm 1.50$ \\
\hline $\begin{array}{l}\text { Duration } \\
\text { since } \\
\text { diagnosis, } \\
\text { years }\end{array}$ & & $0.14 \pm 0.40$ & $0.20 \pm 0.53$ & $0.20 \pm 0.45$ \\
\hline $\begin{array}{l}\text { Stage of } \\
\text { disease (H\&Y) }\end{array}$ & & $1.46 \pm 0.50$ & $1.56 \pm 0.50$ & $1.56 \pm 0.50$ \\
\hline $\begin{array}{l}\text { Total MDS- } \\
\text { UPDRS Part III } \\
\text { (motor) }\end{array}$ & & $19.47 \pm 9.16$ & $21.11 \pm 8.69$ & $21.38 \pm 8.89$ \\
\hline $\begin{array}{l}\text { MoCA } \\
\text { examination }\end{array}$ & $28.38 \pm 1.23$ & $27.40 \pm 2.19^{*}$ & $27.07 \pm 2.63^{*}$ & $27.30 \pm 2.31$ * \\
\hline GDS score & $1.30 \pm 2.14$ & $2.16 \pm 2.45^{*}$ & $2.50 \pm 2.43^{*}$ & $2.61 \pm 2.61$ * \\
\hline $\mathrm{GDS} \geq 5$ & $11(6.2 \%)$ & $23(11.2 \%)^{*}$ & $17(16.5 \%)^{*}$ & $16(18.0 \%)^{*}$ \\
\hline RBDSQ & $2.97 \pm 2.38$ & $4.35 \pm 2.86^{*}$ & $4.29 \pm 2.50^{*}$ & $5.02 \pm 3.18^{*}$ \\
\hline Gait Index & - & $0.32 \pm 0.80$ & $0.32 \pm 0.57$ & $0.40 \pm 0.69$ \\
\hline
\end{tabular}

Significant difference compared with healthy controls ( $\left.{ }^{*} p<0.05\right)$. GDS, Geriatric Depression Score; H\&Y, Hoehn \& Yahr scale; MDS-UPDRS, Movement Disorder Society Unified Parkinson's Disease Rating Scale; MoCA, Montreal Cognitive Assessment; PD-BC, bilaterally reduced caudate binding;PD-NC, no reduced ${ }^{123} \mathrm{I}$-FP-CIT binding in either caudate; PD-UC, reduced ${ }^{123} \mathrm{I}-\mathrm{FP}-\mathrm{CIT}$ binding in one caudate only; RBD, REM sleep behaviour disorder; RBDSQ, RBD Screening Questionnaire. 
Follow-up

Complete 4-year clinical assessments were available for 323 patients with PD and SPECT imaging for 267. Follow-up clinical data were available for 151 HCs. Given the smaller PD sample at follow-up, we compared the variables of gender, age, MoCA scores, GDS scores, RBDSQ scores and caudate SBRs between patients with and without available data at follow-up in order to check whether the follow-up cohort was representative of the baseline one. We did not find significant differences in these variables (online supplementary table 3).

We then compared ratings and frequencies of cognitive impairment, depressive features, RBD and gait problems at the 4-year follow-up among the three PD subgroups as classified at baseline according to their caudate SBRs. Since age and mean putamen SBR at baseline were significantly different across the three groups, we adjusted the comparisons of continuous scores for these variables. Finally, we analysed the occurrence of caudate dysfunction at follow-up and the conversion to atypical parkinsonism.

\section{Cognitive impairment}

At 4-year follow-up, MoCA scores were significantly different across PD subgroups: the PD-BC group had significantly lower MoCA scores compared with the PD-NC group (25.05 \pm 4.32 vs $26.93 \pm 3.06, \mathrm{p}<0.001)$ and to the PD-UC group $(25.05 \pm 4.32$ vs $27.16 \pm 3.21, \mathrm{p}<0.001$ ) (figure $2 \mathrm{~A}$ ). After adjusting for age, years of education and baseline mean putamen SBR, these
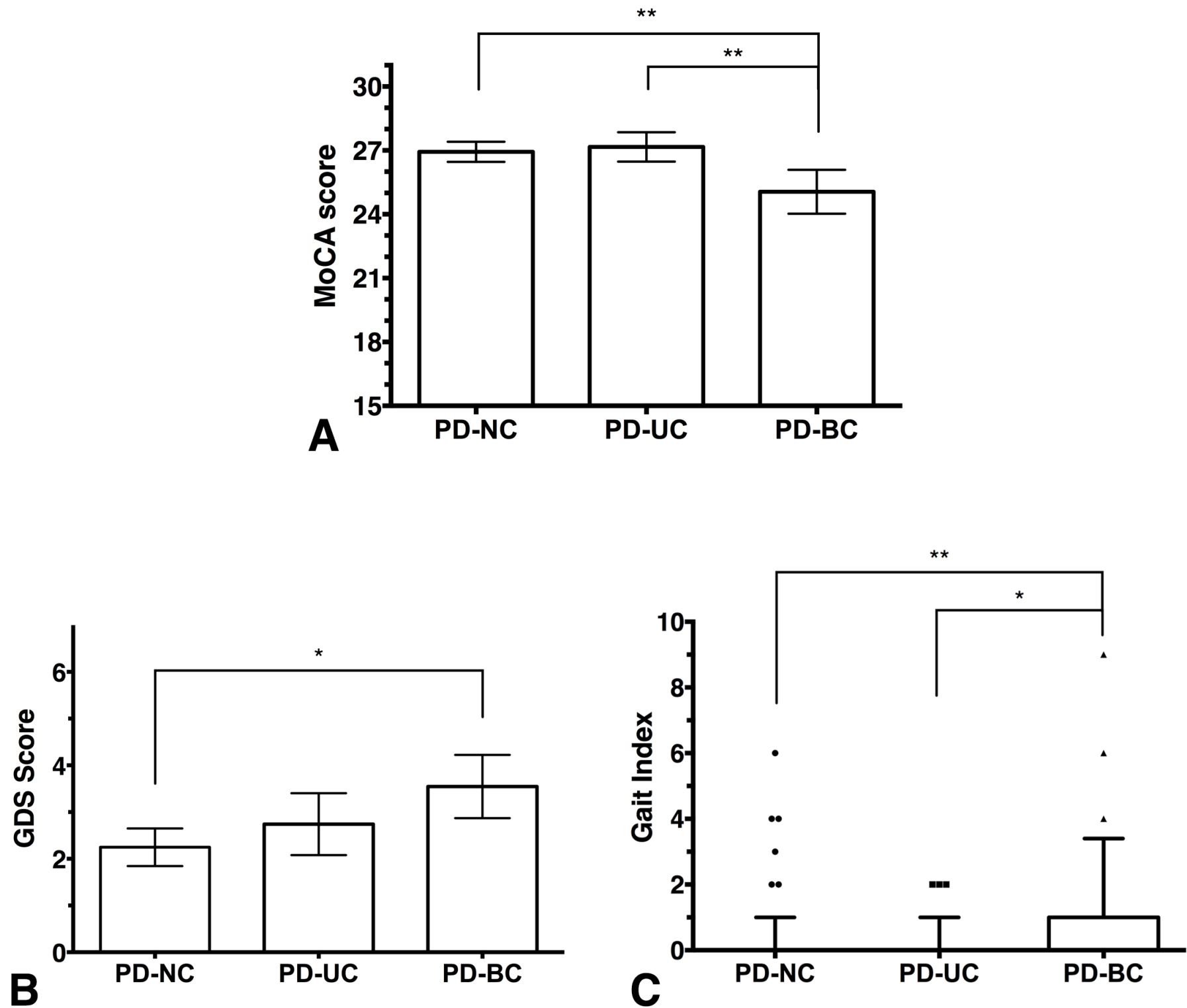

Figure 2 Boxplots showing differences in outcomes in PD subgroups ( $n=323)$ at the 4-year follow-up. (A) MoCA, (B) GDS and (C) Gait Index score. PD-NC: Parkinson's disease patients with no reduced caudate ${ }^{123} \mathrm{I}-\mathrm{FP}-\mathrm{CIT}$ binding compared with the controls' mean at baseline. PD-UC: Parkinson's disease patients with reduced baseline caudate ${ }^{123}$ I-FP-CIT binding below -2 SDs of the controls' mean in one caudate only. PD-BC: Parkinson's disease patients with reduced baseline caudate ${ }^{123} \mathrm{I}-\mathrm{FP}-\mathrm{CIT}$ binding below -2 SDs of the controls' mean in both caudate nuclei. (A) Bar charts representing mean MoCA scores with 95\% Cls at the 4-year follow-up. (B) Bar charts representing mean GDS scores with 95\% Cls at the 4-year follow-up. (C) Boxes and whiskers representing distributions of the gait index scores (product of MDS-UPDRS II subitems 2.12 and 2.13) at the 4-year follow-up. Boxes: 25th to 75th percentiles; whiskers 95th percentile. ${ }^{*} P<0.05,{ }^{* *} p<0.001$. GDS, Geriatric Depression Score; MoCA, Montreal Cognitive Assessment score; PD, Parkinson's disease. 
differences retained statistical significance. We further analysed the relative contributions of baseline caudate and putamen to MoCA scores by carrying out a univariate analysis holding baseline caudate and putamen SBRs as covariates: only caudate SBR was significantly associated with MoCA scores $(B=1.522$, $\mathrm{p}<0.01$; putamen: $\mathrm{B}=-0.747, \mathrm{p}=0.483$ ).

Furthermore, cognitive impairment as determined by the investigator based on the clinical interview, the presence of significant functional impairment, and review of neuropsychological testing, was much more common in the whole cohort of patients with PD compared with HCs $(21.8 \%$ vs $5 \%, \mathrm{p}<0.001)$. Across the three PD subgroups, the PD-BC patients were significantly more likely to develop cognitive impairment $(42.3 \%$, $\left.\chi^{2}=23.04, p<0.001\right)$ compared with PD-UC patients $(16.7 \%)$ and PD-NC (15.1\%).

\section{Mood disorders}

At 4-year follow-up, the PD-BC group showed significantly higher GDS scores compared with PD-NC after adjusting for age and mean putamen SBR $(3.40 \pm 3.01$ vs $2.25 \pm 2.63, \mathrm{p}<0.05)$; no significant differences were observed between PD-BC and PD-UC groups and between PD-NC and PD-UC groups (figure 2B). Again, we conducted a general linear model (GLM) univariate analysis in order to test whether baseline caudate and putamen SBRs were associated with GDS scores: only caudate binding was significantly associated with GDS scores ( $\mathrm{B}=-0.999, \mathrm{p}<0.05$; putamen: $\mathrm{B}=0.532, \mathrm{p}=0.544)$.

In terms of clinically significant depression, the PD-BC group had a higher frequency compared with the PD-NC group $(29.6 \%$ vs $\left.10.8 \%, \chi^{2}=12.67, \mathrm{p}<0.001\right)$ but the frequency in the PD-UC group $(21.2 \%)$ did not differ significantly from that of the other two groups.

RBD

At follow-up, there was no significant difference in RBD scores between PD subgroups.

\section{Gait impairment}

The PD-BC group showed more severe gait impairment compared with the PD-NC group (mean rank 135.31 vs 112.02, $\mathrm{U}=4735, \mathrm{p}<0.001$ ) and to the PD-UC group (mean rank 86.57 vs $72.75, \mathrm{U}=2516, \mathrm{p}<0.05)$. No significant differences were found between PD-NC and PD-UC (figure 2C).

\section{Progression of caudate involvement and conversion to atypical parkinsonism}

Of 267 patients with SPECT imaging available at 4-year follow-up, at baseline 134 were classified as PD-NC (50.2\%), 74 as PD-UC (27.7\%) and 59 PD-BC (22.1\%). After 4 years, 67.9\% of the baseline PD-NC group progressed to have either unilateral or bilateral caudate involvement; of the baseline PD-UC group, $78.4 \%$ progressed to a bilateral involvement. Overall, at follow-up, $83.9 \%$ of the population showed significantly reduced caudate DAT availability (unilateral 22.5\%, bilateral $61.4 \%$ ), while $16.1 \%$ still had a bilateral caudate SBR within -2 SDs of the controls' mean (table 2 and figure $1 \mathrm{~B}$ ).

The percentage of decline in caudate ${ }^{123}$ I-FP-CIT binding from baseline to follow-up was similar in the three groups $(\mathrm{PD}-\mathrm{NC}=-24.5 \pm 13.0 \%, \mathrm{PD}-\mathrm{UC}=-23.8 \pm 16.4 \%, \mathrm{PD}-\mathrm{BC}=-$ $25.1 \pm 17.2 \% ; \mathrm{F}=1.38, \mathrm{p}=0.253)$. In the whole $\mathrm{PD}$ cohort, the rates of decline in the four nuclei analysed were: right putamen (mean $\% \pm S D$ ) 28.36\% $25.94 \%$; left putamen:
Table 2 Degree of caudate involvement in 267 patients with an available DATscan at 4-year follow-up and relationship to original PD subgroups

\begin{tabular}{lllll}
\hline & $\begin{array}{l}\text { No caudate } \\
\text { involvement }\end{array}$ & $\begin{array}{l}\text { Unilateral } \\
\text { caudate } \\
\text { involvement } \\
\text { at 4 years }\end{array}$ & $\begin{array}{l}\text { Bilateral } \\
\text { caudate } \\
\text { involvement } \\
\text { at 4 years } \\
(\boldsymbol{n}, \%)\end{array}$ & $\begin{array}{l}\text { Total } \\
\text { number } \\
\text { of }\end{array}$ \\
$\begin{array}{l}\text { Baseline PD } \\
\text { subgroups }(\mathbf{n}=267)\end{array}$ & $\begin{array}{l}(\boldsymbol{n}, \%) \\
\text { patients }\end{array}$ \\
\hline $\begin{array}{l}\text { PD-NC group } \\
(n, \%)\end{array}$ & $43(32.1 \%)$ & $44(32.8 \%)$ & $47(35.1 \%)$ & 134 \\
$\begin{array}{l}\text { PD-UC group } \\
(n, \%)\end{array}$ & - & $16(21.6 \%)$ & $58(78.4 \%)$ & 74
\end{tabular}

$(n, \%)$

\begin{tabular}{|c|c|c|c|c|}
\hline $\begin{array}{l}\text { PD-BC group } \\
(n, \%)\end{array}$ & - & - & $59(100 \%)$ & 59 \\
\hline $\begin{array}{l}\text { Total } \\
(n, \%)\end{array}$ & $43(16.1 \%)$ & $60(22.5 \%)$ & $164(61.4 \%)$ & 267 \\
\hline
\end{tabular}

Percentages and total number of patients are relative to each row. PD-BC, bilaterally reduced caudate binding; PD-NC, no reduced ${ }^{123} \mathrm{I}-\mathrm{FP}-\mathrm{CIT}$ binding in either caudate; PD-UC, reduced ${ }^{123}$ I-FP-CIT binding in one caudate only.

$28.28 \% \pm 27.67 \%$; right caudate: $24.64 \% \pm 17.84 \%$; left caudate: $25.09 \% \pm 19.09 \%$.

Of the eight patients whose diagnosis changed to an atypical parkinsonism, at baseline six presented with bilaterally reduced caudate (three with MSA, three with DLB), while two had a bilaterally normal caudate SBR (one with MSA, one with CBD; their follow-up SPECT scans were not available). Baseline caudate mean SBR of these eight patients were significantly lower than the remaining patients $(U=722.5, p<0.01)$; however, individual values overlapped with patients with idiopathic PD (data not shown).

\section{DISCUSSION}

In this longitudinal study, we analysed the occurrence of significant early caudate dopaminergic dysfunction, as defined by ${ }^{123}$ I-FP-CIT SBR below -2 SDs compared with the controls' mean, in the large PPMI cohort of patients with recently diagnosed PD. Furthermore, we studied the association between this early caudate DAT loss and the presence or subsequent development of cognitive impairment, depressive features, RBD and gait problems. Unlike previous studies, we provided a clinically viable method to define patients at risk of developing such symptoms. In fact, software for semiquantitative analysis of DAT-SPECT to estimate DAT availability in striatal subregions (ie, putamen and caudate) are now commercially available and already adopted in many Nuclear Medicine Units. The assessment of the occurrence of early caudate dysfunction as described in this paper can therefore be easily performed in clinical settings and could serve as a risk marker for worse disease progression.

In this study, we have used two SDs below the mean of the control population as the threshold to discriminate between normal or abnormal DAT availability of the caudate. DAT availability declines with age and our control population is slightly younger than the PD-BC group. Therefore, in order to assess if there was a significant age-related decline in caudate DAT availability in our control cohort, we compared mean caudate SBRs in three subgroups of controls classified according to their age $(50-59,60-69, \geq 70)$. No significant differences were found in mean caudate DAT binding across these groups, suggesting that age-related decline of caudate binding in our cohort is minimal.

Previous neuroimaging and pathophysiological studies on striatal dopamine loss have shown uneven patterns of dopamine depletion in the basal ganglia. ${ }^{14-16}$ This has led to the concept of 
a posterior-to-anterior gradient in early PD, with an asymmetric reduced striatal binding in the posterior putamen and relative preservation in the head of caudate. In this study, we observed baseline unilateral or bilateral caudate dopaminergic dysfunction, reflected by a reduced ${ }^{123}$ I-FP-CIT binding $<-2$ SDs compared with controls, in nearly half $(48.4 \%)$ of this early, untreated PD cohort. This finding indicates that early caudate dopaminergic dysfunction is not uncommon in patients with PD at the onset of parkinsonian symptoms, as opposed to what was previously thought. Patients with unilateral and bilateral caudate involvement had a significantly reduced putamen ${ }^{123}$ I-FP-CIT binding compared with patients with no caudate dysfunction; however, the caudate/putamen ratio was not significantly different across PD subgroups, suggesting that the posterior-to-anterior gradient of dopaminergic loss is substantially preserved in all patients with PD.

At follow-up, the majority of patients (61.4\%) showed significantly reduced DAT availability in both caudate nuclei, and $22.5 \%$ had significant reduction in one caudate only. Only $16.1 \%$ of patients retained normal caudate ${ }^{123}$ I-FP-CIT uptake bilaterally. These findings are in keeping with previous studies that have demonstrated similar caudate and putaminal involvement in patients with longer disease duration. ${ }^{17} 18$

Our results did not show a significant difference between the three PD subgroups, defined by the level of caudate dysfunction, on baseline assessments of cognition, mood or gait but only found a difference between PD subgroups with these measures at the 4-year follow-up. It is likely that baseline caudate dopaminergic deficits are not severe enough to cause marked impairment in either cognition, mood or gait but later predispose to such impairments, possibly in combination with other neurotransmitter systems dysfunction. Indeed, we interestingly found that this early, absolute significant reduction of caudate signal on ${ }^{123}$ I-FP-CIT SPECT compared with controls at the time of clinical diagnosis is associated with worse outcomes in regard to cognitive impairment, affective symptoms and gait problems at the 4-year follow-up.

Several SPECT studies using dopaminergic tracers have proposed a role for caudate dysfunction in cognitive impairment associated with PD. ${ }^{3424}$ Furthermore, three studies have reported early caudate dopaminergic dysfunction as a predictor for future cognitive impairment. ${ }^{5-7}$ Our analysis showed that patients with PD with baseline bilateral caudate DAT binding reductions, but not unilateral reductions, are at greater risk of developing cognitive impairment and having lower MoCA scores regardless of their putamen DAT availability, age and years of education. Therefore, in comparison with the previously mentioned studies, we propose a practical approach that could be used in the clinical practice at time of diagnosis to determine which patients are at increased risk of developing cognitive problems in a near future.

Depression is highly prevalent in PD with rates as high as $40 \%{ }^{26}$ The aetiology of depression has been attributed to deficits, with varying degrees, in the dopaminergic, serotonergic, cholinergic and noradrenergic pathways. ${ }^{27}$ Several neuroimaging studies have shown an association between reduced DAT availability in the striatum and depressive symptoms. ${ }^{28-30}$ One study demonstrated a negative association between right caudate nucleus ${ }^{123}$ I-FP-CIT binding and the severity of depressive symptoms. ${ }^{8}$ Our study suggests that early bilateral caudate dopaminergic dysfunction is associated with an increased frequency of clinically significant depression and to worse depressive symptoms, regardless of age and mean putamen SBR. Therefore, our results concur with other studies and provide further evidence that depressive symptoms in patients with PD may be associated with dopaminergic denervation of the caudate. Again, we provided a clinical neuroimaging marker to determine which patients are at increased risk of developing worse depressive symptoms.

Our study also indicated that early bilateral caudate involvement may predispose to future development of self-reported gait impairment. A previous PET study has demonstrated substantial caudate nucleus dopaminergic denervation in patients with PD with gait difficulty which was predominantly associated with the right caudate. ${ }^{31}$

We did not identify significant differences in RBDSQ scores across the three groups of patients at baseline or at the 4-year follow-up. Although one study found lower DAT availability in the caudate nucleus of patients with $\mathrm{PD}$ with RBD compared with those without RBD, ${ }^{9}$ it is plausible that a more extensive network involving other neurotransmitter systems, such as the cholinergic pathways, ${ }^{32}$ are responsible for these symptoms. Furthermore, while the RBDSQ is a valid and widely used tool to assess RBD symptoms, only polysomnography, which was not performed in the PPMI study, could have provided a complete evaluation of RBD manifestations.

Eight patients initially diagnosed with idiopathic PD had their diagnosis switched to an atypical parkinsonism (MSA or CBD) or to DLB at the 4-year follow-up. These eight patients showed caudate mean SBR significantly lower than patients with idiopathic PD; however, individual values overlapped between the two groups. This is in line with previous studies that have demonstrated that in atypical parkinsonisms ${ }^{123}$ I-FP-CIT SPECT generally shows lower striatum uptake and different uptake patterns but is unable to accurately distinguish those syndromes from idiopathic PD. ${ }^{33} 34$

A number of limitations should be addressed. At follow up we studied a smaller sample size of 323 patients with clinical assessments and 267 patients with available SPECT scans; however, these are still relatively considerable numbers. In order to test whether the baseline and follow-up cohorts were similar, we verified that age, gender, MoCA scores, GDS scores, RBDSQ scores and caudate SBR at baseline were not significantly different between patients included and excluded from the follow-up analysis(online supplementary material 1). The clinical follow-up analysis only included the imaging variables of caudate and putamen DAT availability; the symptoms analysed might presumably be caused by extensive networks involving more than one neurotransmitter system; therefore, future multitracer studies may be necessary to confirm and further examine our results. Where appropriate, that is, for cognitive impairment and depressive symptoms scales scores, we corrected the analysis for the known variables of putamen SBR, age and years of education. The gait index variable was not normally distributed; therefore, we were unable to carry out a corrected GLM as for the other scales ratings.

\section{CONCLUSION}

In this study, we have demonstrated a high frequency of early caudate dopaminergic dysfunction in patients with recently diagnosed PD. Furthermore, our findings suggest that caudate quantification of DAT availability shortly after diagnosis may have an important role in identifying patients at risk of clinical progression to cognitive impairment, depression and gait problems in the near future. In fact, early bilateral ${ }^{123}$ I-FP-CIT caudate uptake below -2 SDs of the controls' mean may be a valid indicator of more rapid onset of such symptoms. This approach might allow 
better prediction of disease course for patients with early PD and could also provide the potential to stratify cases for early targeted disease-modifying therapies.

Acknowledgements We thank John Seibyl, Heather Ovens and the Neuroimaging team at Invicro, a Konica Minolta Company (New Haven, Connecticut) for their valuable support in the Imaging analysis. Data used in the preparation of this article were obtained from the Parkinson's Progressive Markers Initiative (PPMI) database (https://www.ppmi-info.org); for up to date information on the study, visit https:// www.ppmi-info.org.

Contributors Study conception and design: JP, RD, NP. Data analysis organisation and execution: JP, RD, NP. Writing of the manuscript: JP, RD, LW, MGS, LR, DJB, DB, NP. Critical revision of the manuscript: LR, DJB, DB, NP.

Funding No funding was provided for the analysis reported in this study. The Parkinson's Progression Markers Initiative - a public- private partnership —is funded by the Michael J. Fox Foundation for Parkinson's Research and funding partners including AbbVie, Allergan, Avid, Biogen, BioLegend, Bristol-Myers Squibb, Celgene, Denali Therapeutics, GE Healthcare, Genentech, GlaxoSmithKline, Lilly, Lundbeck, Merck, Meso Scale Discovery, Pfizer, Piramal, Prevail Therapeutics, Roche, Sanofi Genzyme, Servier, Takeda, Teva, UCB, Verily, Voyager Therapeutics and Golub Capital.

Competing interests None declared.

Patient consent for publication Not required.

Ethics approval All participating PPMI sites received approval from an ethical standards committee prior to study initiation and written informed consent for research was obtained from all participants in the study.

Provenance and peer review Not commissioned; externally peer reviewed.

Data availability statement Data are available on reasonable request.

Open access This is an open access article distributed in accordance with the Creative Commons Attribution Non Commercial (CC BY-NC 4.0) license, which permits others to distribute, remix, adapt, build upon this work non-commercially, and license their derivative works on different terms, provided the original work is properly cited, appropriate credit is given, any changes made indicated, and the use is non-commercial. See: http://creativecommons.org/licenses/by-nc/4.0/.

\section{REFERENCES}

1 Marié RM, Barré L, Dupuy B, et al. Relationships between striatal dopamine denervation and frontal executive tests in Parkinson's disease. Neurosci Lett 1999;260:77-80.

2 Rinne JO, Portin R, Ruottinen $\mathrm{H}$, et al. Cognitive impairment and the brain dopaminergic system in Parkinson disease: [18F]fluorodopa positron emission tomographic study. Arch Neurol 2000;57:470-5

3 Müller U, Wächter T, Barthel H, et al. Striatal [123I]beta-CIT SPECT and prefronta cognitive functions in Parkinson's disease. J Neural Transm 2000;107:303-19.

4 Nobili F, Campus C, Arnaldi D, et al. Cognitive-nigrostriatal relationships in de novo, drug-naïve Parkinson's disease patients: A [I-123]FP-CIT SPECT study. Mov. Disord. 2010;25:35-43.

5 Arnaldi D, Campus C, Ferrara M, et al. What predicts cognitive decline in de novo Parkinson's disease? Neurobiology of Aging 2012;33:1127.e11-1127.e20.

6 Schrag A, Siddiqui UF, Anastasiou Z, et al. Clinical variables and biomarkers in prediction of cognitive impairment in patients with newly diagnosed Parkinson's disease: a cohort study. The Lancet Neurology 2017;16:66-75.

7 Caspell-Garcia C, Simuni T, Tosun-Turgut D, et al. Multiple modality biomarker prediction of cognitive impairment in prospectively followed de novo Parkinson disease. Plos One 2017;12:e0175674.

8 Vriend C, Raijmakers P, Veltman DJ, et al. Depressive symptoms in Parkinson's disease are related to reduced [123I]FP-CIT binding in the caudate nucleus. Journal of Neurology, Neurosurgery \& Psychiatry 2014;85:159-64.

9 Arnaldi D, De Carli F, Picco A, et al. Nigro-caudate dopaminergic deafferentation: a marker of REM sleep behavior disorder? Neurobiology of Aging 2015:36:3300-5.
10 Zhang L, Li T-N, Yuan Y-S, et al. The neural basis of postural instability gait disorder subtype of Parkinson's disease: a PET and fMRI study. CNS Neurosci Ther 2016;22:360-7.

11 Ouchi Yet al. Changes in dopamine availability in the nigrostriatal and mesocortical dopaminergic systems by gait in Parkinson's disease. Brain 2001;124:784-92.

12 Kish SJ, Shannak K, Hornykiewicz O. Uneven pattern of dopamine loss in the striatum of patients with idiopathic Parkinson's disease. N Engl J Med 1988;318:876-80.

13 Fearnley JM, Lees AJ. Ageing and Parkinson's disease: substantia nigra regional selectivity. Brain 1991;114:2283-301.

14 Winogrodzka Aet al. [123I]beta-CIT SPECT is a useful method for monitoring dopaminergic degeneration in early stage Parkinson's disease. J Neurol Neurosurg Psychiatry 2003;74:294-8.

15 Seibyl JP, Marek KL, Quinlan D, et al. Decreased single-photon emission computed tomographic [123I]beta-CIT striatal uptake correlates with symptom severity in Parkinson's disease. Ann Neurol 1995:38:589-98.

16 Innis RB, Seibyl JP, Scanley BE, et al. Single photon emission computed tomographic imaging demonstrates loss of striatal dopamine transporters in Parkinson disease. Proceedings of the National Academy of Sciences 1993;90:11965-9.

17 Nandhagopal R, Kuramoto L, Schulzer M, et al. Longitudinal progression of sporadic Parkinson's disease: a multi-tracer positron emission tomography study. Brain 2009;132:2970-9.

18 Nurmi E, Ruottinen HM, Bergman J, et al. Rate of progression in Parkinson's disease: a 6-[18F]fluoro-L-dopa PET study. Mov Disord 2001;16:608-15.

19 Marek K, Jennings D, Lasch S, et al. The Parkinson progression marker Initiative (PPMI). Progress in Neurobiology 2011;95:629-35.

20 Nasreddine ZS, Phillips NA, BÃ@dirian ValÃ@rie, et al. The Montreal Cognitive assessment, MoCA: a brief screening tool for mild cognitive impairment. J Am Geriatr Soc 2005:53:695-9.

21 Almeida OP, Almeida SA. Short versions of the geriatric depression scale: a study of their validity for the diagnosis of a major depressive episode according to ICD-10 and DSM-IV. Int. J. Geriat. Psychiatry 1999;14:858-65.

22 Emre M, Aarsland D, Brown R, et al. Clinical diagnostic criteria for dementia associated with Parkinson's disease. Mov Disord. 2007;22:1689-707.

23 Litvan I, Goldman JG, Tröster Al, et al. Diagnostic criteria for mild cognitive impairment in Parkinson's disease: movement disorder society Task Force guidelines. Mov. Disord. 2012;27:349-56.

24 Ekman U, Eriksson J, Forsgren L, et al. Functional brain activity and presynaptic dopamine uptake in patients with Parkinson's disease and mild cognitive impairment: a cross-sectional study. The Lancet Neurology 2012;11:679-87.

25 Pellecchia MT, Picillo M, Santangelo G, et al. Cognitive performances and DAT imaging in early Parkinson's disease with mild cognitive impairment: a preliminary study. Acta Neurol Scand 2015;131:275-81.

26 Burn DJ. Beyond the iron mask: towards better recognition and treatment of depression associated with Parkinson's disease. Mov Disord. 2002;17:445-54.

27 Aarsland D, Påhlhagen S, Ballard CG, et al. Depression in Parkinson diseaseepidemiology, mechanisms and management. Nat Rev Neurol 2012;8:35-47.

28 Rektorova I, Srovnalova H, Kubikova R, et al. Striatal dopamine transporter imaging correlates with depressive symptoms and Tower of London task performance in Parkinson's disease. Mov Disord. 2008;23:1580-7.

29 Weintraub D, Newberg AB, Cary MS, et al. Striatal dopamine transporter imaging correlates with anxiety and depression symptoms in Parkinson's disease. J Nucl Med 2005; 46:227-32

30 Hesse S, Meyer PM, Strecker K, et al. Monoamine transporter availability in Parkinson's disease patients with or without depression. Eur J Nucl Med Mol Imaging 2009:36:428-35.

31 Bartels AL, de Jong BM, Giladi N, et al. Striatal dopa and glucose metabolism in PD patients with freezing of gait. Mov Disord. 2006:21:1326-32.

32 Kotagal V, Albin RL, Müller MLTM, et al. Symptoms of rapid eye movement sleep behavior disorder are associated with cholinergic denervation in Parkinson disease. Ann Neurol. 2012:71:560-8.

33 Pirker W, Asenbaum S, Bencsits G, et al. [123I]?-CIT spect in multiple system atrophy, progressive supranuclear palsy, and corticobasal degeneration. Mov. Disord. 2000;15:1158-67.

34 Varrone A, Marek KL, Jennings D, et al. [123I]?-CIT SPECT imaging demonstrates reduced density of striatal dopamine transporters in Parkinson's disease and multiple system atrophy. Mov Disord. 2001;16:1023-32. 